\title{
Methods of sample size calculation in descriptive retrospective burden of illness studies
}

\author{
Karissa M. Johnston ${ }^{1,2^{*}}$, Pardis Lakzadeh', Bonnie M. K. Donato ${ }^{3}$ and Shelagh M. Szabo ${ }^{1}$
}

\begin{abstract}
Background: Observational burden of illness studies are used in pharmacoepidemiology to address a variety of objectives, including contextualizing the current treatment setting, identifying important treatment gaps, and providing estimates to parameterize economic models. Methodologies such as retrospective chart review may be utilized in settings for which existing datasets are not available or do not include sufficient clinical detail. While specifying the number of charts to be extracted and/or determining whether the number that can feasibly extracted will be clinically meaningful is an important study design consideration, there is a lack of rigorous methods available for sample size calculation in this setting. The objective of this study was to develop recommended sample size calculations for use in such studies.

Methods: Calculations for identifying the optimal feasible sample size calculations were derived, for studies characterizing treatment patterns and medical costs, based on the ability to comprehensively observe treatments and maximize precision of resulting 95\% confidence intervals. For cost outcomes, if the standard deviation is not known, the coefficient of variation $\mathrm{c} v$ can be used as an alternative. A case study of a chart review of advanced melanoma (MELODY) was used to characterize plausible values for $\mathrm{cv}$ in a real-world example.
\end{abstract}

Results: Across sample sizes, any treatment given with greater than $1 \%$ frequency has a high likelihood of being observed. For a sample of size 200, and a treatment given to $5 \%$ of the population, the precision of a $95 \%$ confidence interval $(\mathrm{Cl})$ is expected to be \pm 0.03 . For cost outcomes, for the median $\mathrm{cv}$ value observed in the MELODY study (0.72), a sample size of approximately 200 would be required to generate a $95 \% \mathrm{CI}$ precise to within $\pm 10 \%$ of the mean.

Conclusion: This study presents a formal guidance on sample size calculations for retrospective burden of illness studies. The approach presented here is methodologically rigorous and designed for practical application in real-world retrospective chart review studies.

Keywords: Pharmacoepidemiology, Epidemiology, Public health

\section{Background}

Observational burden of illness studies are widely used to characterize treatment patterns, resource utilization, costs, and clinical outcomes associated with a disease. In pharmacoepidemiology, burden of illness studies can contextualize the current treatment setting, identify important treatment gaps and their associated consequences,

* Correspondence: kjohnston@broadstreetheor.com

${ }^{1}$ Broadstreet Health Economics and Outcomes Research, 343 Railway St Vancouver BC, Vancouver V6A 1A4, Canada

${ }^{2}$ Memorial University, St John's, Newfoundland, Canada

Full list of author information is available at the end of the article characterize the potential benefits of a new therapy, and provide estimates to parameterize economic models [1-3].

With the increased availability of "big data", methodological considerations for observational research often focus on the use of large databases - while challenges remain in this setting, available sample size and power do not tend to be problematic given the large pool of individuals from which to draw [4]. However, such databases are not able to answer all burden of illness research questions, due to insufficient clinical detail

(c) The Author(s). 2019 Open Access This article is distributed under the terms of the Creative Commons Attribution 4.0 International License (http://creativecommons.org/licenses/by/4.0/), which permits unrestricted use, distribution, and reproduction in any medium, provided you give appropriate credit to the original author(s) and the source, provide a link to the Creative Commons license, and indicate if changes were made. The Creative Commons Public Domain Dedication waiver (http://creativecommons.org/publicdomain/zero/1.0/) applies to the data made available in this article, unless otherwise stated. 
being recorded, the rarity of the condition, or an appropriate database not being available for a jurisdiction of interest. In this setting, chart review remains a powerful methodology for accessing comprehensive data directly from de-identified patient charts - allowing for real-world burden of illness parameters to be assessed, for example, for individuals with a particular range of laboratory measures or a specific genetic biomarker.

An important limitation to chart review is feasibility of achieving a sufficiently large sample at a reasonable cost. Depending on the setting, charts may not be centrally linked, requiring engagement of individual clinicians to obtain chart access; and if detailed medical data are required to meet study objectives, data extraction may involve several hours per chart. Thus, in the context of a retrospective chart review, a critical a priori consideration is the number of charts that are required to meaningfully address research questions, and the feasibility of identifying and extracting this number of charts. Conversely, researchers may know at the outset how many charts are available to be extracted, and must consider the expected value of the information, before deciding whether to perform chart extraction.

While methodological guidance for conducting retrospective chart reviews has been previously described, formal guidance on sample size calculations is not available $[5,6]$. Commonly-used sample size calculations are typically based on a hypothesis-testing framework. Since the outcomes of burden of illness studies tend to be descriptive rather than inferential, such calculations are not aligned with burden of illness objectives, and have limited relevance in this setting. For a burden of illness study the aim of sample size calculation is to ensure sufficient precision in descriptive outcomes, e.g. characterized by the width of $95 \%$ confidence intervals (CIs). Given the absence of validated methods for a priori sample size estimates in the context of retrospective chart review studies, the aim of this article was to develop and present rigorous approaches for sample size calculation using a real-world case study.

\section{Methods}

Sample size formulae are presented for parameters that are of frequent interest in the context of a burden of illness study. This includes summarizing treatment patterns (such as the proportion receiving each treatment, and a comprehensive list of therapies used for a condition, including rare therapies), presence of comorbidities, clinical outcomes such as laboratory measures or calculated disease scores, and resource utilization and cost outcomes (including results stratified by subgroup). In practice, multiple outcomes are often of interest and sample size calculations will generate different projections across outcomes; sample size considerations can be focussed on the outcomes of greatest interest, and/or those that generate the largest sample size requirement. The methods described here are generally applicable to categorical (treatment patterns, comorbidity proportion) and continuous outcomes (costs, continuous clinical measures); treatment patterns and costs, respectively, are used as illustrative examples.

\section{Sample size calculations for categorical outcomes} (e.g. treatment patterns)

When considering treatment distributions in a population, assuming a binomial distribution $(n, p)$ for receiving a particular treatment, in which the $n$ represents the sample size and $p$ represents the probability of receiving the treatment, the following are direct results of the binomial distribution:

Expected number of observed patients receiving the treatment is:

$$
n \times p
$$

The width of the $95 \% \mathrm{CI}$ for estimating $p$ is:

$$
\pm 1.96 \times \sqrt{\frac{p(1-p)}{n}}
$$

The probability of not receiving the treatment is $(1-p)$, and therefore the probability of all $n$ patients not receiving the treatment is $(1-p)^{n}$, such that the probability of observing at least one patient receiving the treatment is:

$$
1-(1-p)^{n}
$$

These formulae can be used to define a sample size that ensures all key treatments will be observed, and that the proportions can be estimated within desired precision. To utilize them to generate sample size requirements, limited a priori data are required;. $n$ can be selected to yield acceptable values for both quantities. Because the required sample size $n$ increases as $p$ moves further from $0.50, p$ can be defined to be the most extreme proportion that would be of interest (e.g. a rare treatment given to $1 \%$ of the population). Alternatively, sample size requirements can be determined for a range of values of $p$, or, in situations where the maximum sample size is fixed due to other constraints, the corresponding minimum treatment frequency can be calculated.

\section{Sample size calculations for continuous outcomes (e.g. costs)}

When considering medical costs, assuming a normal distribution for mean costs $\mu$ and standard deviation $\sigma$, precision associated with a particular sample size can be characterized by the width $W$ of the $95 \%$ CI: 


$$
W=1.96 \times \frac{\sigma}{\sqrt{n}}
$$

If an estimate of $\sigma$ is available, e.g. based on published evidence for another jurisdiction or a similar indication, then the width of the CI can be expressed for the maximum feasible sample size $n$. Alternatively, Eq. (4) can be rearranged so that the required $n$ can be calculated to obtain $95 \%$ CIs for a desired width $\pm \mathrm{W}$ :

$$
n=\frac{1.96^{2} \sigma}{\mathrm{W}^{2}}
$$

Frequently, estimates of $\sigma$ aren't available, making sample size calculations challenging - a challenge common across a variety of contexts when estimating sample size. In the absence of such data, one option is to consider the coefficient of variation $\mathrm{c}_{\mathrm{v}}$, defined as $\frac{\sigma}{\mu}$. Based on this, $\sigma$ can be expressed as $c_{v} \times \mu$, and for assumed values of $c_{v}$ and $\mu, n$ can be estimated without specific estimates for $\sigma$. Replacing $\sigma$ in Eq. (4), the width of a 95\% CI can be expressed as:

$$
\pm 1.96 \times \frac{c_{v} \times \mu}{\sqrt{n}}
$$

Via this formula, required sample size $n$ can be calculated for a desired with W based on rearranging Eq. (6). If an estimate of $\mu$ is available, $n$ can therefore be presented for a desired absolute CI width W Eq. (7). Otherwise, if $\mu$ is unknown, the desired width could instead be expressed as a desired percentage of the mean, e.g. it is desired to estimate mean cost with a $95 \%$ confidence interval precise to within $+/-\mathrm{V} \%$ of the mean. This is equivalent to saying that $\mathrm{V}=\frac{\mathrm{W}}{\mu}$, which can be incorporated into Eq. (7); doing this allows for calculation of $n$ without knowing $\mu$ or $\sigma$ Eq. (8). If $V$ is set equal to $100 \%$, the width of the CI would be equal to $\mu$ so that the lower bound of the CI for costs would be 0 . Smaller values of $\mathrm{V}$, are associated with narrower CIs, entirely above 0 .

Expressed with respect to absolute width W, the required sample size is:

$$
\left(\frac{1.96 \times c_{v} \times \mu}{V}\right)^{2}
$$

Expressed with respect to width $\mathrm{V}$ defined as percentage of the mean, the required sample size is:

$$
\left(\frac{1.96 \times c_{v}}{V}\right)^{2}
$$

A real-world case study is presented below, to describe the precision achieved for a retrospective chart review of burden of illness including treatment patterns and costs in advanced melanoma (the MELODY study) [7], with a total sample size of 655 patients across the United Kingdom (UK), Italy, and France. Costs were presented both per individual overall, as well as per user of specific utilization categories (e.g. hospitalization costs amongst the subgroup with non-zero hospitalization). The range of $\mathrm{c}_{\mathrm{v}}$ ratios observed in the MELODY study are presented to provide plausible values for future studies.

A sample size calculator based on the formulas presented is available as supplementary material (see Additional file 1).

\section{Results}

Based on Eqs. (1-3), Table 1 presents calculated relationships between sample sizes and the expected number of cases to be observed, the probability of observing a treatment in practice, and expected precision, for a range of treatment probabilities. Across sample sizes, any treatment given with greater than $1 \%$ frequency has a high likelihood of being observed. For a sample of size 200 , and a treatment given to $5 \%$ of the population, the precision of a $95 \% \mathrm{CI}$ is expected to be \pm 0.03 ; i.e. the expected 95\% CI would be (0.02-0.08). Generally, with respect to characterizing treatment patterns, sample sizes above 200 are only required for treatments given to $1 \%$ of the population or less, or if particularly narrow precision estimates are needed. The information in Table 1 can be used to identify the optimal sample size based on a treatment pattern-related research question, or, in the case of a fixed sample size, to identify the level of detail that can be described.

For cost estimation, based on Eqs. (7) and (8), assuming that an estimate for the standard deviation is not available, an estimate of $\mathrm{c}_{\mathrm{v}}$ can instead be used. Observed data from the MELODY study are presented in Table 2 to describe a range of $\mathrm{c}_{\mathrm{v}}$ observed in practice. Trends in observed $c_{v}$ values included higher values typically observed for hospice and hospital costs relative to outpatient costs, and higher when considering the full population of included patients vs. the subset with non-zero use of a particular category of utilization. Across all categories considered, values for $c_{v}$ ranged from 0.26 to 4.30 , with a median value of 0.72 . In practice, a range of possible values for can be considered, based on any a priori knowledge regarding heterogeneity in the population with respect to health resource utilization and cost outcomes of interest, e.g. the expected range of disease severity, and the anticipated distribution of costs with respect to routine maintenance and care vs. high cost acute treatment such as inpatient stays.

Required sample sizes based on Eq. (8) are presented in Fig. 1. Figure 1a displays sample size requirement for the full range of values for $c_{v}$ observed in the MELODY 
Table 1 Expected number of observed cases; probability and expected precision of observing a treatment in practice

\begin{tabular}{lllllll}
\hline \multicolumn{7}{c}{$\begin{array}{l}\text { Expected number of individuals receiving treatment; (Probability of observing treatment at least once) } \pm \text { Expected 95\% confidence interval } \\
\text { width for proportion receiving treatment }\end{array}$} \\
\cline { 2 - 7 } & $n=50$ & $n=100$ & $n=200$ & $n=300$ & $n=500$ & $n=1000$ \\
\hline$p=0.01$ & $1(0.39) ; \pm 0.03$ & $1(0.63) ; \pm 0.02$ & $2(0.87) ; \pm 0.01$ & $3(0.95) ; \pm 0.01$ & $5(0.99) ; \pm 0.01$ & $10(1.00) ; \pm 0.01$ \\
$p=0.05$ & $3(0.92) \pm 0.06$ & $5(0.99) \pm 0.04$ & $10(1.00) \pm 0.03$ & $15(1.00) \pm 0.02$ & $25(1.00) \pm 0.02$ & $50(1.00) \pm 0.01$ \\
$p=0.10$ & $5(0.99) \pm 0.08$ & $10(1.00) \pm 0.06$ & $20(1.00) \pm 0.04$ & $30(1.00) \pm 0.03$ & $50(1.00) \pm 0.03$ & $100(1.00) \pm 0.02$ \\
$p=0.25$ & $13(1.00) \pm 0.12$ & $25(1.00) \pm 0.08$ & $50(1.00) \pm 0.06$ & $75(1.00) \pm 0.05$ & $125(1.00) \pm 0.04$ & $250(1.00) \pm 0.03$ \\
$p=0.50$ & $25(1.00) \pm 0.14$ & $50(1.00) \pm 0.10$ & $100(1.00) \pm 0.07$ & $150(1.00) \pm 0.06$ & $250(1.00) \pm 0.04$ & $500(1.00) \pm 0.03$ \\
$p=0.75$ & $28(1.00) \pm 0.12$ & $75(1.00) \pm 0.08$ & $150(1.00) \pm 0.06$ & $225(1.00) \pm 0.05$ & $375(1.00) \pm 0.04$ & $750(1.00) \pm 0.03$ \\
\hline
\end{tabular}

study, while Fig. 1b only considers values for $\mathrm{c}_{\mathrm{v}}$ between 0 and 1 . For the median $\mathrm{c}_{\mathrm{v}}$ value of 0.72 from the MELODY study, a sample size of approximately 200 would be required to generate a $95 \% \mathrm{CI}$ precise to within $\pm 10 \%$ of the mean. For a $\mathrm{c}_{\mathrm{v}}$ of 4.5 , more than 8000 individuals would be required to estimate a $95 \%$ CI precise to within $\pm 10 \%$ of the mean. Thus, in situations where large variability is anticipated, e.g. a sample ranging from zero costs to long and costly hospitalizations, the required sample size may be prohibitively large for a chart review or prospective study, requiring access to an administrative or other large database.

When determining optimal sample size, it is important to consider any subgroups of interest, and either target such subgroups directly in sampling strategy or account for expected sample size needs if only the overall population is to be sampled. For example, if descriptive cost analyses are to be conducted for a sample that is estimated to be $20 \%$ of the overall population, and a required sample size is identified based on required precision for this subgroup, the full sample will need to be $1 / 20 \%=5 x$ the required sample size identified by the subgroup.

\section{Discussion}

Chart reviews are commonly used to assess clinical outcomes, treatment patterns, and healthcare resource use and costs for more rare health conditions, for very specific indications, or in cases where the required parameters are not captured in large datasets. Despite their ubiquity, methodologic guidance on how to conduct rigorous chart reviews, particularly with respect to selecting appropriate sample sizes, are few. Even the existing methodologic guidance on how to conduct chart reviews provide little direction in this area; suggesting it is beyond the scope of the guidance [6], or assuming a randomized trial-like, hypothesis testing framework [5], rather than methods appropriate for the descriptive objectives that often frame chart reviews. We therefore aimed to fill this gap by providing a framework for estimating appropriate sample sizes for study designs that aim to precisely estimate treatment patterns or resource use parameters, rather than comparing outcomes between groups. These can be especially relevant for situations where the availability of patient charts is limited by time, budget, or the available population size.

We found that for objectives around summarizing categorical and continuous, sample sizes of 100 patients and greater are in most cases sufficient; although larger samples may be required to characterize cost outcomes and/or examine subgroups. For objectives around cost estimation, greater sample sizes are required, particularly if relatively precise estimation is desired, and/or if results specific to particular subgroups are of interest. If 200 to 400 patient charts is the maximum feasible sample size, as is often the case in practice, it can be expected that

Table 2 Observed values of coefficient of variation $c_{v}$ from the MELODY study

\begin{tabular}{|c|c|c|c|c|c|c|c|c|c|}
\hline & \multicolumn{3}{|c|}{ United Kingdom } & \multicolumn{3}{|l|}{ Italy } & \multicolumn{3}{|l|}{ France } \\
\hline & $\overline{M e a n}$ & SD & $C_{v}$ & Mean & SD & $C_{v}$ & Mean & SD & $C_{v}$ \\
\hline \multicolumn{10}{|l|}{ Costs per person } \\
\hline Hospitalization & 3225 & 7132 & 2.21 & 2486 & 10,689 & 4.3 & 6262 & 6553 & 1.05 \\
\hline Hospice & 2394 & 4247 & 1.77 & 185 & 396 & 2.14 & 298 & 511 & 1.72 \\
\hline Outpatient & 587 & 275 & 0.47 & 29 & 15 & 0.51 & 28 & 31 & 1.11 \\
\hline \multicolumn{10}{|l|}{ Costs per user } \\
\hline Hospitalization & 11,437 & 13,432 & 1.17 & 3306 & 2209 & 0.67 & 11,469 & 8859 & 0.77 \\
\hline Hospice & 10,363 & 5103 & 0.49 & 185 & 94 & 0.51 & 3429 & 2079 & 0.61 \\
\hline Outpatient & 782 & 314 & 0.4 & 72 & 28 & 0.39 & 59 & 15 & 0.26 \\
\hline
\end{tabular}




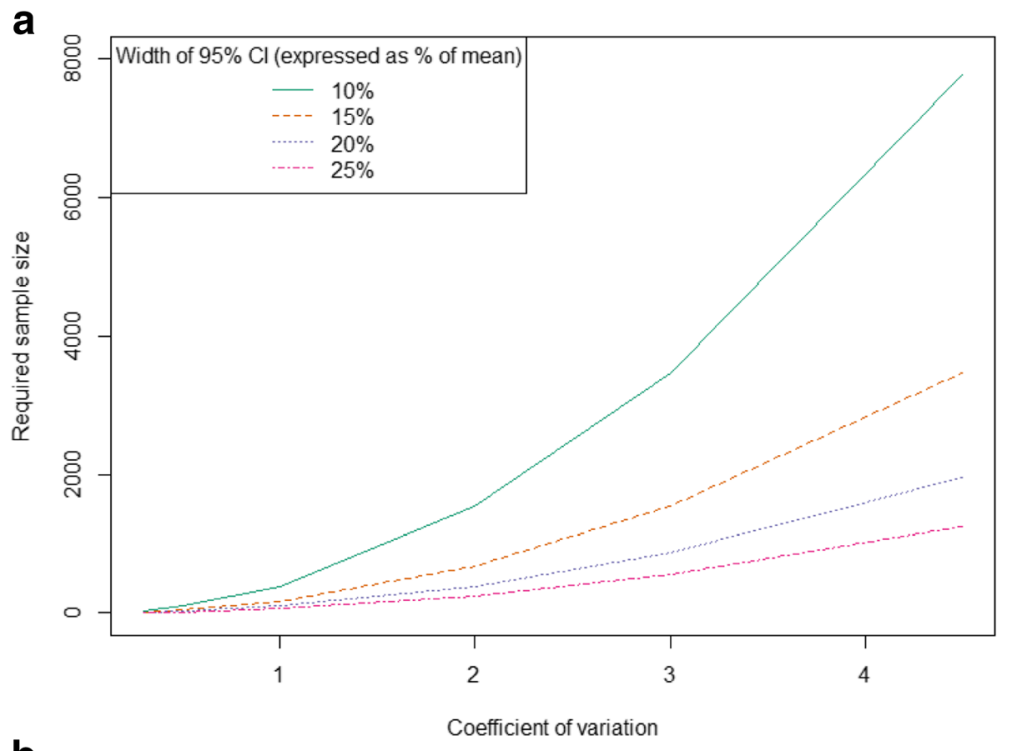

b

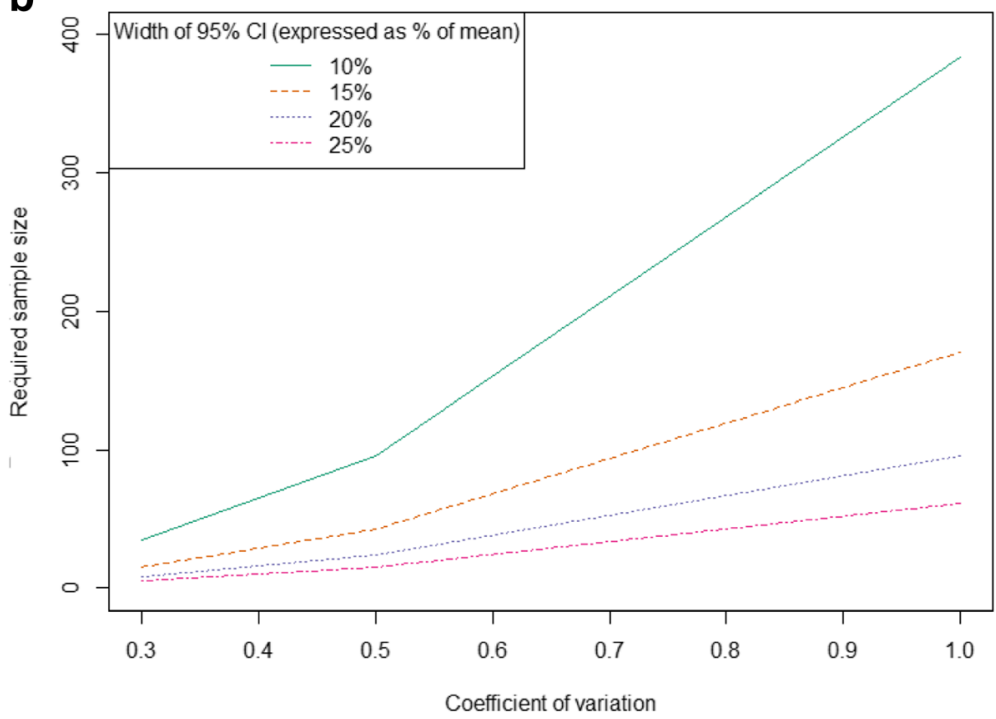

Fig. 1 Sample size required across values for coefficient of variation from (a) 0-4.5 and (b) 0-1.0

cost estimate $95 \%$ confidence intervals will be precise to within $5-15 \%$ of the mean, depending on the ratio of standard deviation to mean costs.

These sample size formulae can be used in two distinct ways. First, if study resources are flexible and desired precision is known, formulae can be used to guide sample size selection. Second, if study resources or available sample size is fixed, formulae can be used to generate anticipated values of precision. We have validated the use of these formulae in a number of other chart review studies [8-10]; and have provided this calculator available online for others to use going forward.

This methodological study addresses an important knowledge gap, as sample sizes are frequently determined using ad-hoc approaches and/or based only on feasibility considerations. Indeed, a non-systematic review of ten recently-published chart reviews focusing on assessing treatment patterns and costs revealed that no studies presented a rationale for their chosen sample size [11-20]. One rule of thumb that has been suggested [6], that is analogous to sample size considerations for regression analyses, is that a minimum of 5-10 charts per variable is required to obtain results that are likely to be both true and clinically useful [21, 22]. However, the number and complexity of outcomes that tend to be considered within a chart review may ultimately limit the utility of this rule in these circumstances, compared to a regression model evaluating the association between a set of independent variables and one dependent variable. 
While the methods presented here are broadly applicable to categorical and continuous variables, this does not span the full range of potential outcomes in descriptive burden of illness studies, as resource utilization variables may be analyzed as count data. While a future extension of this work could include formulae based on appropriate distributions (Poisson, negative binomial), the formulas presented for continuous outcomes can be used to generate approximate results based on a normal approximation to the Poisson distribution. For cost data, skewness due to large numbers of zero responses and a few large outlying values may limit the appropriateness of normality assumption; in practice, methods such as two-stage models and/ or functional transformations may be undertaken when analyzing data [23]. In sample size estimation, while the assumption of normality for potentially non-normal data is a limitation, using $\mathrm{c}_{\mathrm{v}}$ values taken from actual cost data will reflect the full scope of variability when such outliers are included and as such are not expected to underestimate required sample size. If doing so yields an infeasibly large sample size, and if statistical techniques are expected to be undertaken at the analysis stage, then this can be incorporated into sample size estimation, e.g. by estimating $\mathrm{C}_{\mathrm{v}}$ based on a sample with zero-value data points excluded, and/or based on log-transformed data.

The strength of this approach is that it presents a simple, straightforward, validated method for estimating sample size for retrospective studies focusing on multiple descriptive outcomes. In chart reviews, these calculations can be useful during the study design phase, to understand the trade- offs between the expenses in time and money from collecting data from additional charts, versus the additional precision around the estimates that can be obtained. This can be particularly important when considering chart review data as inputs for economic models, where the variability around the estimate can have a major impact. As is the case with estimating sample size a priori for any type of outcome, the applicability of these formulas is limited by the availability of useful preliminary data to use as the basis for the calculations.

A priori estimates of sample size are required when designing chart reviews and other retrospective studies with study objectives that focus on describing treatment patterns, resource use, and costs. However, validated and easily-implemented methods to estimate sample size in this situation are not readily available, or frequently used. The approach presented here is methodologically rigorous and designed for practical application in real-world retrospective chart review studies.

\section{Conclusion}

This study presents a formal guidance on sample size calculations for retrospective burden of illness studies.
The approach presented here is methodologically rigorous and designed for practical application in real-world retrospective chart review studies and can be used in two distinct ways; where [1] the study resources are flexible and desired precision is known, formulas can be used to guide sample size selection, or [2] if study resources or available sample size is fixed, formulas can be used to generate anticipated values of precision.

\section{Additional file}

Additional file 1: Sample size calculator. The authors have provided an Excel sample size calculator as supplementary material (Additional file 1) to help guide editors and reviewers through the approach presented. If the article is published, the authors are interested in expanding the Excel calculator to an interactive website to facilitate use of the methods by other researchers. (XLSX $15 \mathrm{~kb}$ )

\section{Abbreviations \\ Cl: Confidence Intervals; UK: United Kingdom}

\section{Acknowledgements}

None.

\section{Funding}

No funding has been received for this study.

Availability of data and materials

Data generated or analysed during this study are included in this published article (and its supplementary information files).

\section{Authors' contributions}

KMJ wrote the first draft. PL, BMKD, and SMZ contributed significantly with comments and suggestions for improvement of the manuscript. KMJ programmed and constructed the sample size calculator included as Additional file 1. All authors have reviewed and accepted the final draft. All authors read and approved the final manuscript.

Ethics approval and consent to participate

Not applicable.

Consent for publication

Not applicable.

\section{Competing interests}

The authors declare that they have no competing interests.

\section{Publisher's Note}

Springer Nature remains neutral with regard to jurisdictional claims in published maps and institutional affiliations.

\section{Author details}

${ }^{1}$ Broadstreet Health Economics and Outcomes Research, 343 Railway St Vancouver BC, Vancouver V6A 1A4, Canada. ' Memorial University, St John's, Newfoundland, Canada. ${ }^{3}$ Alexion Pharmaceuticals, New Haven, CT, USA.

Received: 29 March 2018 Accepted: 28 December 2018

Published online: 09 January 2019

\section{References}

1. Worster A, Haines T. Advanced statistics: understanding medical record review (MRR) studies. Acad Emerg Med Off J Soc Acad Emerg Med. 2004;11(2):187-92.

2. Chandran A, Schaefer C, Ryan K, Baik R, McNett M, Zlateva G. The comparative economic burden of mild, moderate, and severe fibromyalgia: results from a retrospective chart review and cross-sectional survey of 
working-age U.S. adults. Journal of managed care pharmacy : JMCP. 2012;18(6):415-26.

3. Lennert B, Farrelly E, Sacco P, Pira G, Frost M. Resource utilization in children with tuberous sclerosis complex and associated seizures: a retrospective chart review study. J Child Neurol. 2013;28(4):461-9.

4. Raghupathi W, Raghupathi V. Big data analytics in healthcare: promise and potential. Health Inform SciSys. 2014;2(1):3.

5. Vassar M, Holzmann M. The retrospective chart review: important methodological considerations. J Educ Eval Health Prof. 2013;10:12.

6. Gearing RE, Mian IA, Barber J, Ickowicz A. A methodology for conducting retrospective chart review research in child and adolescent psychiatry. J Can Acad Child Adolesc Psychiatry. 2006;15(3):126-34

7. Lebbe C, Lorigan P, Ascierto P, et al. Treatment patterns and outcomes among patients diagnosed with unresectable stage III or IV melanoma in Europe: a retrospective, longitudinal survey (MELODY study). Eur J Cancer. 2012:48(17):3205-14.

8. Szabo Smo KM, Qatami L, Korenblat Donato BM, Korol EE, Al Madani AA, Al Awadi FF, Al-Ansari J, Maclean R, Levy AR. Quality of Care for Patients with type 2 diabetes mellitus in Dubai: a HEDIS-like assessment. Int J Endocrinol. 2015;2015(8).

9. Osenenko KMS SM, Qatami L, Korenblat Donato BM, Al Madani A A, Al Awadi FF, Al-Ansari J, Maclean R, Levy AR. Patterns of Care and treatment target success among persons with type 2 diabetes Mellitus in Dubai: a retrospective cohort study. Value Health. 2015;7:87-93.

10. Johnston $\mathrm{K}$, Levy AR, Lorigan $\mathrm{P}$, et al. Economic impact of healthcare resource utilisation patterns among patients diagnosed with advanced melanoma in the United Kingdom, Italy, and France: results from a retrospective, longitudinal survey (MELODY study). Eur J Cancer. 2012;48(14):2175-82.

11. Corbisiero R, Buck DC, Muller D, Bharmi R, Dalal N, Kazemian P. What is the cost of non-response to cardiac resynchronization therapy? Hospitalizations and healthcare utilization in the CRT-D population. J Interv Card Electrophysiol. 2016;47(2):189-95.

12. Ritchie BM, Sylvester KW, Reardon DP, Churchill WW, Berliner N, Connors JM. Treatment of heparin-induced thrombocytopenia before and after the implementation of a hemostatic and antithrombotic stewardship program. J Thromb Thrombolysis. 2016;42(4):616-22.

13. Stewart ML, Biddle M, Thomas T. Evaluation of current feeding practices in the critically ill: a retrospective chart review. Intensive Crit Care Nurs. 2016; 38:24-30.

14. Edwards PK, Hadden KB, Connelly JO, Barnes CL. Effect of Total joint arthroplasty surgical day of the week on length of stay and readmissions: a clinical pathway approach. J Arthroplast. 2016;31(12):2726-9.

15. Selva-Sevilla C, Gonzalez-Moral ML, Tolosa-Perez MT. The psychiatric patient as a health resource consumer: costs associated with electroconvulsive therapy. Front Psychol. 2016;7:790.

16. Ho MY, Renouf DJ, Cheung WY, et al. Patterns of practice with third-line anti-EGFR antibody for metastatic colorectal cancer. Current oncology (Toronto, Ont). 2016;23(5):329-33.

17. Doyle Strauss L, Weizenbaum E, Loder EW, Rizzoli PB. Amitriptyline dose and treatment outcomes in specialty headache practice: a retrospective cohort study. Headache. 2016;56(10):1626-34.

18. Shah SS, Satin AM, Mullen JR, Merwin S, Goldin M, Sgaglione NA. Impact of recent guideline changes on aspirin prescribing after knee arthroplasty. J Orthop Surg Res. 2016;11(1):123.

19. Emery P, Solem C, Majer I, Cappelleri JC, Tarallo M. A European chart review study on early rheumatoid arthritis treatment patterns, clinical outcomes, and healthcare utilization. Rheumatol Int. 2015;35(11):1837-49.

20. Abdelnabi M, Patel A, Rengifo-Pardo M, Ehrlich A. Insurance coverage of biologics for moderate-to-severe psoriasis: a retrospective, observational 5-year chart review. Am J Clin Dermatol. 2016:17(4):421-4.

21. Sackett DLH, BR, Guyatt GH, Tugwell P. Clinical epidemiology: a basic science for clinical medicine. Second edition. Lippincott Williams and Wilkins; 1991.

22. Raykov T, Widaman KF. Issues in applied structural equation modeling research. Struct Equ Model Multidiscip J. 1995;2(4):289.

23. Mihaylova B, Briggs A, O'Hagan A, Thompson SG. Review of statistical methods for analysing healthcare resources and costs. Health Econ. 2011;20(8):897-916.

\section{Ready to submit your research? Choose BMC and benefit from}

- fast, convenient online submission

- thorough peer review by experienced researchers in your field

- rapid publication on acceptance

- support for research data, including large and complex data types

- gold Open Access which fosters wider collaboration and increased citations

- maximum visibility for your research: over $100 \mathrm{M}$ website views per year

At BMC, research is always in progress.

Learn more biomedcentral.com/submissions 